\title{
Cosmological Perturbations on a Bouncing Brane
}

\author{
Robert Brandenberger ${ }^{*}$ Hassan Firouzjahi $\left.\right|^{\dagger}$ and Omid Sarem周 \\ Physics Department, McGill University, \\ 3600 University Street, Montreal, Canada, H3A 2T8
}

\begin{abstract}
The cosmological perturbations on a bouncing brane are studied. The brane is moving inside a Klebanov-Strassler throat where the infra-red region of the geometry is smoothly cut off. For an observer confined to the world-volume of the brane, this results in a non-singular bouncing mirage cosmology. We have calculated the scalar perturbations corresponding to the normal displacements of the brane. This is performed in the probe brane limit where the gravitational back-reaction of the brane on the bulk throat is absent. Our model provides a framework for studying the transfer of fluctuations from a contracting to an expanding phase. We find that the spectral index of the dominant mode of the metric fluctuation is un-changed, unlike what is obtained by gluing contracting to expanding Einstein universes with the help of the usual matching conditions. Assuming that the fluctuations start off in a vacuum state on sub-Hubble scales during the contracting phase, it is shown that the resulting spectral index $n_{s}$ on super-Hubble scales in the expanding phase has a large blue tilt. When the brane is moving slowly inside the throat and its kinetic energy is negligible compared to its rest mass, one finds $n_{s}=3$. For a fast-rolling brane with a large kinetic energy, the spectral index is $n_{s}=2.3$. This may put severe constraints on models of mirage cosmology.
\end{abstract}

Keywords : D-brane, Bouncing cosmology

PACS numbers:

*Electronic address: rhb@physics.mcgill.ca

†Electronic address: firouz@physics.mcgill.ca

${ }^{\ddagger}$ Electronic address: omid@physics.mcgill.ca 


\section{INTRODUCTION}

Recent cosmological observations provide an increasingly clear picture of the current structure of the universe on large scales [1]. The universe is spatially flat to high accuracy, the background cosmology is homogeneous and isotropic, and there is a superimposed spectrum of almost scale-invariant, nearly Gaussian, and nearly adiabatic cosmological fluctuations. A viable theory of early universe cosmology must explain these observations. Inflationary cosmology [2] by far is the best model currently available which can explain these data. Furthermore, it successfully solves the flatness, homogeneity and horizon problems of Standard Big Bang (SBB) cosmology. On the other hand, current realizations of inflation are plagued by various conceptual problems [3]. Two of the problems which motivate this study are the presence of an initial singularity [4] which signals an incompleteness of the background cosmology, and the "Trans-Planckian" problem, namely the fact that the fluctuations emerge from sub-Planckian wavelengths and hence from a zone of ignorance about the fundamental physics [3, 5]. One should thus be open-minded towards alternative scenarios which may, in principle, be able to explain the data while avoiding the shortcomings associated with inflationary cosmology.

Bouncing cosmologies can solve the horizon problem of the SBB cosmology. In bouncing cosmologies, each period of expansion is preceded by a period of contraction during which the co-moving Hubble radius is decreasing while the horizon continues to increase. Hence, in a bouncing universe the horizon can easily be made to be larger than the past light cone at the time of last scattering, the region over which the universe is seen to be homogeneous and isotropic. The entropy or size problem of SBB cosmology also disappears in the context of a bouncing cosmology: if the universe begins large in a phase of contraction, there is no reason why the initial entropy should be small. The flatness problem of SBB cosmology, however, persists (see e.g. [6]).

A key question which determines the viability of any cosmological model with a bounce concerns the spectrum of cosmological perturbations. It is an extremely non-trivial challenge to find a scenario which is consistent with the current data. There are two issues involved. First, one must find a mechanism for producing an almost scale-invariant spectrum, and secondly, one must be able to evolve the spectrum through the bounce region.

Recently, there have been several attempts to construct bouncing cosmologies, in particu- 
lar the Pre-Big-Bang [7] and the Ekpyrotic [8] scenarios. Both, however, involve a singularity at the bounce point which prevents a consistent computation of the spectrum of fluctuations. (In a new version of the Ekpyrotic scenario, the singularity can be smoothed out by invoking ghost condensation [9].) There have been attempts to construct non-singular bouncing cosmologies, making use of the interplay of curvature and matter with wrong sign kinetic terms [10], quintom matter [11], k-essence [12] and higher derivative gravity actions [13, 14]. An elegant way of obtaining a non-singular bouncing cosmology is by means of "mirage cosmology" [15].

In this paper, we study the evolution of fluctuations through a non-singular bounce in the context of mirage cosmology. We focus mainly on two questions. First, does the spectral index of the cosmological fluctuations change when passing through this bounce? Secondly, does a scale-invariant spectrum emerge if we set up the fluctuations in a vacuum state early in the period of contraction. The answer to the second question is "no". Concerning the first question, we find that the spectrum goes through the bounce without change in the spectral index. This result is very interesting. In work on Pre-Big-Bang and Ekpyrotic cosmology where the contracting phase and the expanding phases were each described by dilaton or Einstein gravity and connected at the singularity by matching conditions [16, 17] analogous to the Israel matching conditions [18], it was found [19, 20] that the spectral index changes: the dominant mode of the metric fluctuations in the contracting phase couples only to the decaying mode in the expanding phase. However, the applicability of these matching conditions has been challenged [21] and concrete studies [12, 22, 23] have shown that it is possible that the spectral index does not change if the bounce is smooth (although other studies give differing results [13, 24]). Mirage cosmology provides a simple setting to study the issue of the transfer of cosmological perturbations through a bounce in a clean way.

\section{THE BOUNCING BACKGROUND}

In "mirage cosmology" [15] our universe is a probe D3-brane moving in extra dimensions. Due to the motion of the brane in the extra dimensions, the induced metric on the brane becomes time-dependent. For an observer confined to the brane this results in a cosmological expansion or contraction. In the probe brane limit, we neglect the back-reaction of the moving brane on the background. This is a good approximation when a single brane is 
moving in the background of a large stack of branes or in the background created by a large number of flux quanta.

The bouncing brane in a throat has been studied in [25], [26], [27] and [28]. The throat was taken to be the warped deformed conifold of the Klebanov-Strassler (KS) solution [29], where the infra-read (IR) region of the geometry is smoothly cut off. The ultra-violet (UV) region of the throat is smoothly glued to the bulk of the Calabi-Yau (CY) manifold. The brane sets off from the UV region, moving towards the IR region. For an observer on the brane this corresponds to a contracting phase. At the tip of the throat, where the warp factor is stationary, the induced scale factor becomes stationary. This results in a bounce point. Finally, the brane bounces back to the UV region which corresponds to an expanding period. In order for the scenario to work, then one has to find a way to consistently connect this period to a late time Big Bang cosmology.

One novel feature of this bouncing solution is that it is singularity-free. This is a manifestation of the fact that the IR region is smoothly cut off. Furthermore, in [26] and [27] it is shown that a cyclic cosmology can be achieved by turning on internal angular momenta. With angular momenta turned on, the brane motion is confined between IR and UV region of the throat which results in a cyclic solution.

The metric of the background is given by

$$
d s^{2}=G_{M N} d X^{M} d X^{N}=h(r)^{-1 / 2} \eta_{\mu \nu} d x^{\mu} d x^{\nu}+g(r) d r^{2}+d s_{5}^{2} .
$$

Here, $G_{M N}$ is the background metric, $h(r)$ is the warp factor, $r$ is the radial direction of the throat and $d s_{5}^{2}$ is the metric along the internal angular directions. In what follows, we do not consider the angular motion of the brane and neglect the last term in Eq. (1).

The space-time indices are represented by capital Latin letters $M, N, \ldots$ while the indices used by the observer confined to the brane are represented by $a, b, \ldots$ The brane is equipped with coordinate $\xi^{a}$. In general, when the brane is moving non-uniformly, one cannot use the static gauge and $\left\{\xi^{a}\right\} \neq\left\{x^{\mu}\right\}$. However, the fluctuations along the spatial coordinates of the branes, $\xi^{i}$, are not physical and we can choose $x^{i}=\xi^{i}$ for $i=1,2,3$. The time coordinate on the brane is defined by $\xi^{0}=t$. In the homogeneous limit when the brane is moving uniformly and no perturbations are present on the brane world-volume, we can set $X^{0}=t$.

The action of the probe D3-brane is given by

$$
S=T_{3} \int d^{4} \xi \sqrt{-\left|\gamma_{a b}\right|}+\mu_{3} \int_{\mathcal{M}} d^{4} \xi P\left(C_{(4)}\right) .
$$


Here, the first term is the usual Dirac-Born-Infeld (DBI) term and the second one is the Chern-Simons (CS) term. Also $T_{3}$ and $\mu_{3}$ are the brane tension and charge, respectively, $\gamma_{a b}$ is the induced metric on the brane, $C_{(4)}$ is the background Ramond-Ramond four-form field and $P\left(C_{(4)}\right)$ represents its pull-back onto the world volume $\mathcal{M}$ of the D3-brane. In the following, we consider the motion of a BPS brane and set $\mu_{3}=T_{3}$.

The position of the brane in the extra dimension is parameterized by

$$
X^{M}=X^{M}\left(\xi^{a}\right) .
$$

For a background space-time metric $G_{M N}$ given in Eq. (1), we have

$$
\left.\gamma_{a b}=G_{M N} \frac{\partial X^{M}}{\partial \xi^{a}} \frac{\partial X^{N}}{\partial \xi^{b}} \quad, \quad P\left(C_{(4)}\right)_{t 123}=\frac{\partial X^{0}}{\partial t} C_{(4)}\right)_{0123} .
$$

For the KS background [29], the radial part of the metric and the form of the warp factor is given by (for a detailed review of this background see [30])

$$
g=\frac{2^{1 / 3}}{6}\left(g_{s} M \alpha^{\prime}\right) I(r)^{1 / 2} K(r)^{-2} \quad, \quad h(r)=\left(g_{s} M \alpha^{\prime}\right)^{2} 2^{2 / 3} \epsilon^{-8 / 3} I(r),
$$

where

$$
I(r)=\int_{r}^{\infty} d x \frac{x \operatorname{coth} x-1}{\sinh ^{2} x}(\sinh 2 x-2 x)^{1 / 3} \quad, \quad K(r)=\frac{(\sinh 2 r-2 r)^{1 / 3}}{2^{1 / 3} \sinh r} .
$$

In these expressions, $g_{s}$ is the string coupling, $M$ is the quantum number of the RR-fluxes turned on inside the $S^{3}$ at the bottom of the throat, and $\epsilon^{2 / 3}$ measures the size of this $S^{3}$ in the units of $\alpha^{\prime}$. The warp factor $h(r)$ has a maximum at the bottom of throat $r=0$ and falls-off exponentially at large $r$. The function $g(r)$ has a minimum at $r=0$ and increases like $r^{1 / 2}$ for large $r$. The fact that $h(r)$ and $g(r)$ are non-singular and their first derivatives vanish at $r=0$ is crucial to obtain the bounce solution. In this background, the $\mathrm{RR}$ four-form field is given by

$$
C_{(4)_{0123}}=g_{s}^{-1} h(r)^{-1} \text {. }
$$

At the homogeneous level the brane moves along the radial directions without any perturbation, and, using Eqs. (4) and (7), we obtain

$$
S=\int d^{4} \xi \mathcal{L}=T_{3} \int d^{4} \xi h^{-1}\left(-\sqrt{1-h^{1 / 2} g \dot{r}^{2}}+1\right),
$$


where the relation $X^{0}=t$ was used. Here and in what follows, an over-dot corresponds to a derivative with respect to $t$, the time measured by the observer on the brane.

Integrating the radial Euler-Lagrange equation which follows from this action, one can construct a constant of integration

$$
E=\dot{r} \partial \mathcal{L} / \partial \dot{r}-\mathcal{L}
$$

This yields the following first order equation of motion for the position of brane $r=R(t)$

$$
\dot{R}^{2}+V_{\text {eff }}(R)=0 \quad, \quad V_{\text {eff }}(R)=-\frac{E h^{1 / 2}(2+E h)}{g(1+E h)^{2}} .
$$

It is easy to see that $E>0$ and $V_{\text {eff }}$ is always negative. Thus, the brane can move in the entire region $0<r<\infty$. On the other hand, to get a finite value for Newton constant (finite $M_{P}$ ), the KS throat should be glued smoothly to the bulk of the CY manifold at $r=r_{c}$. This implies that the brane would be moving in the region $0<r<r_{c}$. We restrict our analysis to the throat region of the KS construction.

An observer confined to the D3-brane feels cosmological expansion or contractions, depending on the direction of the brane's motion. For this observer, the four-dimensional metric is given by

$$
d s_{4}^{2}=-d \tau^{2}+a(\tau)^{2} d \mathbf{x}^{2}
$$

where $\mathbf{x}$ denote the three spatial coordinates and the proper time is defined by

$$
d \tau^{2}=-\gamma_{00} d t^{2}=h^{-1 / 2}\left(1-g h^{1 / 2} \dot{R}^{2}\right) d t^{2},
$$

and the scale factor is given by $a(\tau)=h(R(t))^{-1 / 4}$.

The corresponding Friedmann equation is

$$
H^{2}=\frac{1}{16} \frac{h^{\prime 2}}{g h^{2}}\left(E^{2} h^{2}+2 E h\right),
$$

where $H=\frac{1}{a(\tau)} \frac{d a(\tau)}{d \tau}$ is the Hubble constant and ' represents the derivative with respect to $r$.

Fig. 1 shows how the scale factor behaves as a function of $\tau$ and $h$ as a function of $r$ (in $h(r)$ the prefactor has been set to 1 ). When the brane moves from the UV region to the IR region of the geometry, the observer sees a cosmic contraction. At $r=0, h^{\prime}=0$, 

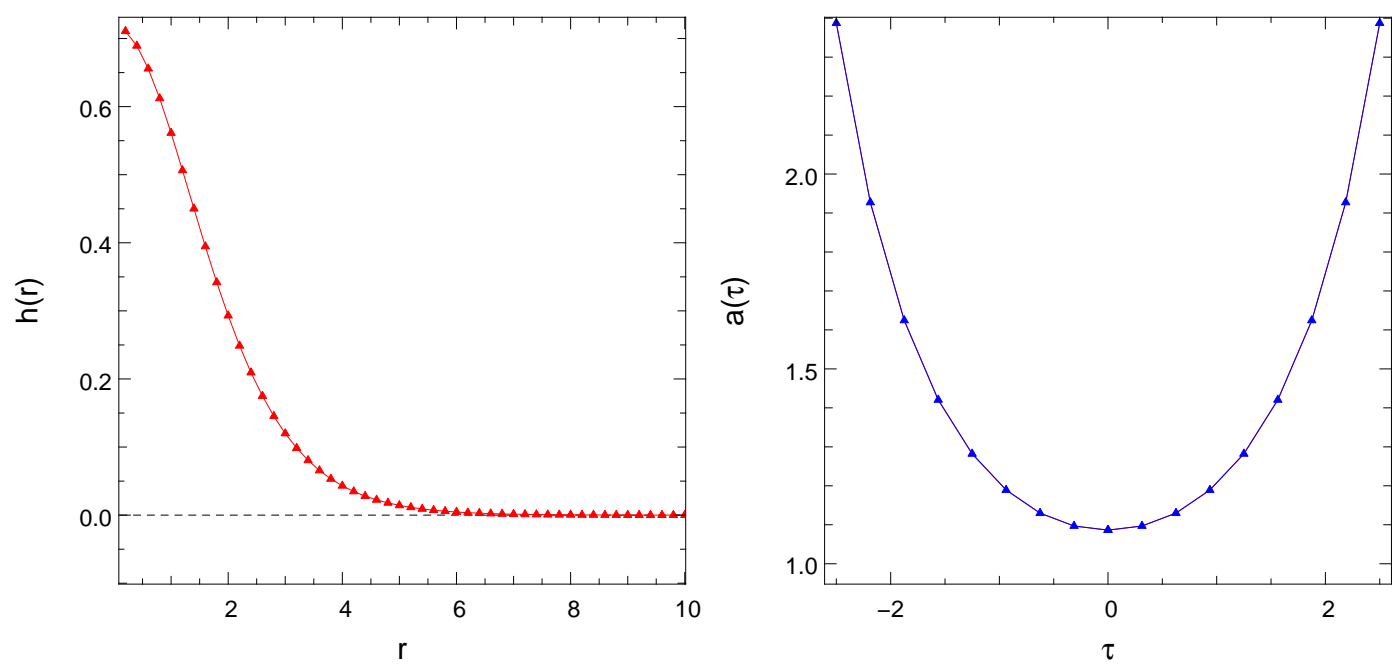

FIG. 1: The warp factor $h(r)$ and the scale factor $a(\tau)$ are presented here. The scale factor is given by $a(\tau)=h(R(t))^{-1 / 4}$. The contracting phase corresponds to the case when the brane moves from the UV region (large $r$ ) towards the IR region ( small $r$ ). The bounce is at $r=0, \tau=0$, where $H=0$. The expanding phase is the mirror image of the contracting phase.

so $H=0$. This corresponds to a bounce point [25]. Afterwards, the brane bounces back towards the UV region and the observer sees a cosmic expansion. The expansion rate $H$ in the expanding phase is the mirror image of the expansion rate in the contracting phase. Using the asymptotic forms of the functions $I(r)$ and $K(r)$ given in Appendix $\mathbf{B}$, one can see that immediately after the bounce, $H$ enters a period of acceleration, followed by a period of deceleration.

To perform the mode analysis in the following sections, it is very helpful to go to conformal time $\eta$, where $a^{2} d \eta^{2}=d \tau^{2}$. Using Eq. (10), we obtain

$$
\left(\frac{d R}{d \eta}\right)^{2}=\frac{h^{1 / 2}}{g} E(2+E h) .
$$

\section{FLUCTUATIONS OF THE BRANE POSITION}

In this section we study the fluctuation of the brane's position while it is moving inside the throat. We are particularly interested in the evolution of these perturbations measured by the observer confined to the brane. Our study is a generalization of what was previously 
studied in [31.

In general, the displacements of the brane can be decomposed into longitudinal and normal components. However, the longitudinal perturbations (which are along the tangential directions of the brane) are not physical and can be gauged away. Suppose $n^{\mu}$ is the spacelike unit normal vector to the brane. Then the normal displacements of the brane are given by

$$
X^{M}=\bar{X}^{M}+\Phi n^{M}
$$

Here, $\Phi$ is the scalar field corresponding to the normal displacement and $\bar{X}^{M}$ represents the brane position at the homogeneous background, i.e. the zeroth order brane position. It is important to note that just considering perturbation along the $r$ direction is not consistent, at least when the probe brane is moving fast. As is clear from (19), the time-like component of the normal vector to the brane becomes important as the probe brane enters into the fast-moving regime(see Fig. 2 ).

At each point, we can span the 5-D space-time by the normal vector base $\left\{e_{a}, n\right\}$, where $\left\{e_{a}\right\}$ are the tangent vector spanning the brane world volume. More, specifically

$$
e_{a}^{M}=\frac{\partial X^{M}}{\partial \xi^{a}} \equiv X_{, a}^{M} \quad, \quad \gamma_{a b}=G\left(e_{a}, e_{b}\right)
$$

where $G($,$) represents the inner product defined by the background metric (1), and \gamma_{a b}$ is the induced metric given in Eq. (4). The normal vector $n^{M}$ is defined by

$$
G\left(n, e_{a}\right)=0 \quad, \quad G(n, n)=1
$$

and the following projection relation holds

$$
\gamma^{a b} X_{, a}^{M} X_{, b}^{N}=G^{M N}-n^{M} n^{N}
$$

For the background defined by Eq. (1), the non-zero components of the normal vector, $n^{0}$ and $n^{r}$, are

$$
n^{0}=g^{1 / 2} h^{1 / 2} \dot{R}\left(1-h^{1 / 2} g \dot{R}^{2}\right)^{-1 / 2} \quad, \quad n^{r}=g^{-1 / 2}\left(1-h^{1 / 2} g \dot{R}^{2}\right)^{-1 / 2} .
$$

Using Eqs. (4) and (7), the action of the moving brane to all order in perturbations is

$$
S=-T_{3} \int d^{4} \xi\left(\sqrt{-|\gamma|}-\frac{\partial X^{0}}{\partial t} C_{(4)}(X)\right) .
$$




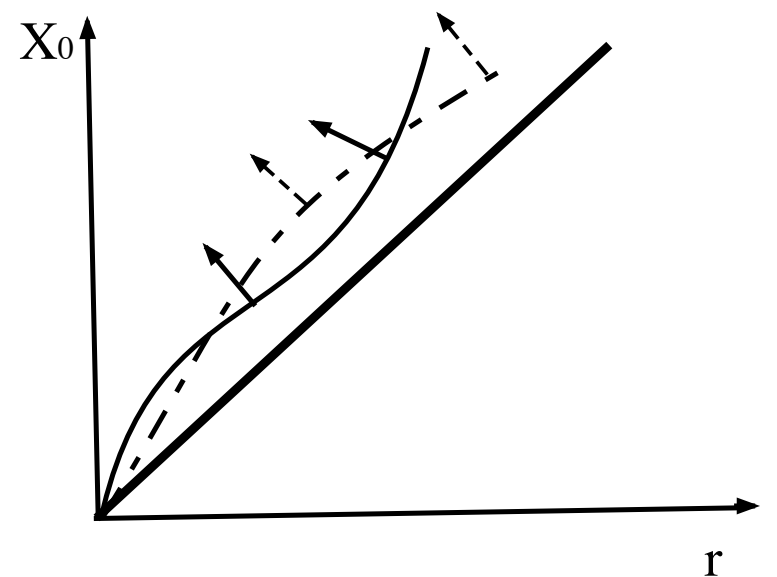

FIG. 2: In this plot the light cone for a fixed point on the brane is plotted. The dashed curved line represents the light-cone of the point when the brane is moving homogeneously governed by Eq. 10. The unit normal vectors are in $X^{0}-r$ plane and have components in both $r$ and $X^{0}$ directions as is evident from Eq. (19). The curved line represents the light-cone when the normal perturbations Eq. 15 are present. Now $n^{M}$ also has components in the tangential directions $X^{i}$.

The equation of motion for $\delta X^{M}$ which follows from this action is obtained in Appendix A, and is

$$
\sqrt{-|\gamma|} K=C_{(4)}^{\prime} n^{r} \frac{\partial X^{0}}{\partial t}-n^{0} \frac{\partial C_{(4)}}{\partial t}
$$

Here, $K$ is the trace of the extrinsic curvature of the surface, $K=\gamma^{a b} K_{a b}$. The extrinsic curvature is defined by

$$
K_{a b}=e_{a}^{M} e_{b}^{N} D_{M} n_{N}=\frac{\partial X^{M}}{\partial \xi^{a}} \frac{\partial X^{N}}{\partial \xi^{b}} D_{M} n_{N}
$$

where $D_{M}$ is the five-dimensional covariant derivative which is compatible with the metric $G_{M N}$. One can check that at the homogeneous level Eq. 21 reproduces the solution studied in the previous section.

To get the linearized equation of motion, we perturb Eq. 21) about the background (15). This is equivalent to expanding the action to second order in the perturbations. We relegate the details of the calculations leading to the perturbed equation of motion to Appendix A and here just give the final result

$$
\nabla^{2} \Phi-M^{2} \Phi=0
$$


where $\nabla^{2}$ is the Laplacian defined by the metric $\gamma_{a b}$ and the effective mass term is given by

$$
M^{2}=-K_{a b} K^{a b}-R_{M N} n^{M} n^{N}+K^{2}+\frac{1}{\sqrt{-|\gamma|}}\left(n^{0}-\frac{n^{r}}{\dot{R}}\right)\left(C_{(4)}^{\prime} n^{r}\right)_{, t} .
$$

In the above expression, $R_{M N}$ is the background Ricci tensor. For the pure AdS background considered in [31] only the first two terms are present and the other two cancel out.

We now calculate the different contributions to $M^{2}$ for our background. From Eq. (21) at the homogeneous level, we find

$$
K=h g^{-1 / 2} C^{\prime}=-\frac{h^{\prime}}{h g^{1 / 2}} .
$$

To calculate the other terms in $M^{2}$, we need to express $n^{0}, n^{r}, \dot{R}$ and $\ddot{R}$ as functions of $r$ and $E$. To do this, we use Eq. 10 which allows $\dot{R}$ to be calculated. Plugging the value of $\dot{R}$ obtained this way into Eq. 19 , one can show that

$$
n^{0}=E^{1 / 2} h^{3 / 4}(2+E h)^{1 / 2} \quad, \quad n^{r}=g^{-1 / 2}(1+E h) .
$$

Using these expressions for $n^{0}$ and $n^{r}$, then for the last term in $M^{2}$ we obtain

$$
\frac{1}{\sqrt{-|\gamma|}}\left(n^{0}-\frac{n^{r}}{\dot{R}}\right)\left(C_{(4)}^{\prime} n^{r}\right)_{, t}=g^{-1 / 2} h\left(h^{\prime} h^{-2} g^{-1 / 2}(1+E h)\right)^{\prime} .
$$

For the term $K^{a b} K_{a b}$, we have

$$
\begin{aligned}
K^{a b} K_{a b} & =\gamma^{a c} \gamma^{b d} K_{a b} K_{c d} \\
& =\gamma^{a c} \gamma^{b d} X_{, a}^{M} X_{, b}^{N} X_{, c}^{P} X_{, d}^{Q} D_{M} n_{N} D_{P} n_{Q} \\
& =D_{M} n_{N} D^{M} n^{N}-D_{M} n_{N} D_{P} n^{N} n^{M} n^{P} \\
& =\frac{h^{\prime 2}}{4 g h^{2}}\left(1+3 E^{2} h^{2}\right) .
\end{aligned}
$$

To go from the second to the third line, the orthogonality condition in Eq. 17 and the projection relation Eq. (18) have been used.

Finally, for the contribution from the Ricci tensor, we get

$$
R_{M N} n^{M} n^{N}=\frac{-1}{8 g^{2} h^{2}}\left[3 E h(2+E h)\left(2 g h^{2}-2 g h h^{\prime}+h h^{\prime} g^{\prime}\right)-8 g h h^{\prime \prime}+4 h h^{\prime} g^{\prime}+10 g h^{2}\right]
$$

Plugging Eqs. 29), 28), 27) and (25) into the expression (24) for $M^{2}$ we obtain

$$
M^{2}=\frac{E}{8 g^{2} h}\left[h(2+3 E h)\left(g^{\prime} h^{\prime}-2 g h^{\prime \prime}\right)+4 g h^{2}\right] .
$$




\section{MODE ANALYSIS}

The differential equation (23) contains a Hubble friction term for $\Phi$. As done in the usual theory of cosmological perturbations (see [32] for an in depth review and [33] for a pedagogical overview), we can extract the effects of the Hubble damping by the field redefinition

$$
\phi(\eta, \vec{x})=a(\eta) \Phi(\eta, \vec{x})=h^{-1 / 4} \Phi
$$

where for convenience we use conformal time $\eta$. Eq. 23) then becomes

$$
\frac{\partial^{2} \phi}{\partial \eta^{2}}-\sum_{i} \partial_{i}^{2} \phi+\left(h^{-1 / 2} M^{2}-\frac{a_{\eta \eta}}{a}\right) \phi=0 .
$$

The above equation is analogous in structure to the equation which describes the evolution of cosmological perturbations in cosmology based on the usual four-dimensional Einstein gravity. On scales larger than the Hubble radius, the negative square mass term $a_{\eta \eta} / a$ is larger than the $k^{2}$ term coming from the spatial gradients. Thus, whereas on sub-Hubble scales the gradient term wins out and leads to the usual micro-physical oscillations, the oscillations freeze out approximately at Hubble radius crossing, and from then on the modes undergo squeezing. As is familiar from the usual theory of cosmological perturbations, scalar metric fluctuations are squeezed not with the factor $a(\eta)$ as would be the case if $M^{2}=0$, but with a modified factor which is usually called $z(\eta)$ which takes into account that $M^{2} \neq 0$.

It is important to point out that, as shown in [31, for the observer on the brane our fluctuation $\Phi$ acts as the usual Bardeen potential [34] which is generally denoted by $\Psi$. In terms of $\Psi$, in longitudinal gauge and in the absence of anisotropic stress, the metric including scalar metric fluctuations takes the form

$$
d s^{2}=-a^{2}(\eta)\left[(1+2 \Psi) d \eta^{2}-(1-2 \Psi) d \mathbf{x}^{2}\right]
$$

It is helpful to work in Fourier space where

$$
\phi_{k}(\eta)=\int d^{3} x \phi(\eta, \vec{x}) e^{-i \vec{k} \cdot \vec{x}}
$$

This transforms Eq. (35) into our desired mode equation

$$
\frac{\partial^{2} \phi_{k}}{\partial \eta^{2}}+\left(k^{2}-v_{e f f}\right) \phi_{k}=0
$$



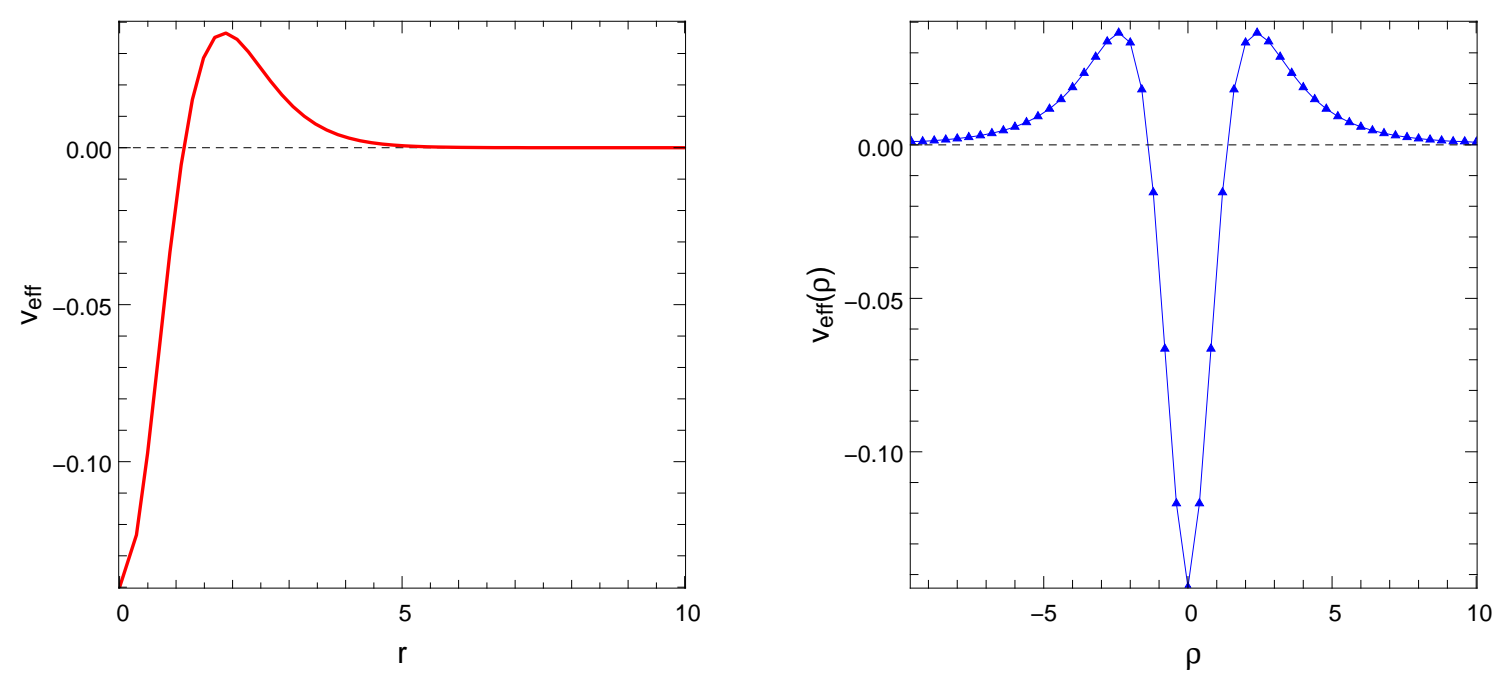

FIG. 3: The plot on the left represents $\tilde{v}_{\text {eff }}$ as a function of $r$. It has a global minimum at $r=0$ with $\tilde{v}_{\text {eff }} \simeq-0.14$ and a maximum at $r \simeq 1.9$ with $\tilde{v}_{\text {eff }} \simeq 0.04$ and exponentially falls off for large $r$. The plot on the right represents $\tilde{v}_{\text {eff }}$ as a function of $\rho=(2 E \beta)^{1 / 2} \tilde{\eta}$. The contracting and the expanding phases are mirror images of each other.

where the "effective potential" is given by

$$
\begin{aligned}
v_{e f f} & =\frac{a_{\eta \eta}}{a}-h^{-1 / 2} M^{2} \\
& =-h^{-1 / 2} M^{2}-\frac{h^{\prime}}{4 h} \frac{\partial^{2} R}{\partial \eta^{2}}-\left(\frac{\partial R}{\partial \eta}\right)^{2}\left(\frac{1}{4} \frac{h^{\prime \prime}}{h}-\frac{5}{16} \frac{h^{\prime 2}}{h^{2}}\right) \\
& =\frac{E^{2} h^{3 / 2}}{8 g}\left(\frac{4 h^{\prime \prime}}{h}-2 \frac{h^{\prime}}{h} \frac{g^{\prime}}{g}+\frac{h^{\prime 2}}{h^{2}}\right),
\end{aligned}
$$

where in the final line Eq. (14) is used to replace $\partial R / \partial \eta$.

We now make an observation which turns out to be important later on. A closer look at (36) reveals that in the vicinity of $r=0, v_{e f f}$ is always negative. This is easily inferred by noting that the 2nd and 3rd terms inside the bracket in (36) are both vanishingly small around $r=0$ to ensure the smoothness of the supergravity background. At the same time the fact that $h$ is a monotonically decreasing function as a function of $r$ with a zero first derivative at $r=0$ guarantees that $h^{\prime \prime} / h$ is negative. So one can conclude that around the bounce point, from the point of view of the brane observer, the effective potential seen by the brane fluctuation is negative.

We try to obtain some analytical insights into the evolution of the mode functions given the effective potential of Eq. (36). Due to the complexity of the KS geometry involving the 
functions $I(r)$ and $K(r)$, it is not possible to have an analytical solution for the entire range of $r$. However, in some limits we can perform analytical calculations which can capture qualitatively (and even quantitatively in the slow probe limit) the numerical results.

Let us define $h(r)=\beta I(r)$ and $g=\gamma I(r)^{1 / 2} K(r)^{-2}$. Notice that Eq. 35 can be rewritten in the following form

$$
\frac{d^{2} \phi_{k}}{d \tilde{\eta}^{2}}+\left(\tilde{k}^{2}-\tilde{v}_{e f f}\right) \phi_{k}=0,
$$

in terms of dimensionless variables

$$
\tilde{\eta} \equiv \frac{E \beta^{3 / 4}}{\gamma^{1 / 2}} \eta \quad, \quad k=\frac{E \beta^{3 / 4}}{\gamma^{1 / 2}} \tilde{k} \quad, \quad v_{e f f}=\frac{E^{2} \beta^{3 / 2}}{\gamma} \tilde{v}_{e f f} .
$$

The potential $\tilde{v}_{\text {eff }}$ is plotted in Fig. 3. As a function of the $r$-coordinate, it has a global minimum at the tip, $r=0$, reaches a global maximum around $r \sim 1.9$, and falls off as $r \exp (-2 r)$ for large values of $r$. In the large $r$ limit, using the asymptotic form of $I(r)$ and $K(r)$ given in Appendix B, one obtains

$$
\tilde{v}_{e f f} \simeq \frac{20 \times 2^{1 / 3}}{6} r e^{-2 r} .
$$

To solve the differential equation (37) analytically, we need to express $\tilde{v}_{e f f}$ as a function of $\tilde{\eta}$. This requires inverting $r$ as a function of $\tilde{\eta}$. The relation between $\tilde{\eta}$ and $r$ is given in Eq. (14), which leads to

$$
\tilde{\eta}= \pm \int_{0}^{r} \frac{d r}{K I^{1 / 2} \sqrt{1+\frac{2}{E \beta I}}} .
$$

The negative branch corresponds to the case when the brane is falling towards the tip of the throat. $\tilde{\eta}=0$ corresponds to the bounce point when the brane reaches the tip. The positive branch corresponds to the case when the brane bounces back towards the UV region of the throat. It is clear that $\tilde{v}_{\text {eff }}$ is symmetric under $\tilde{\eta} \rightarrow-\tilde{\eta}$.

The above integral can not be computed for all value of $r$. However, in the limit of large values of $r$, when the KS solutions is well-approximated by pure AdS geometry, one can compute the integral analytically. Using the asymptotic forms of $I$ and $K$, we have

$$
\begin{aligned}
\tilde{\eta} & \simeq \pm 2^{-1 / 6} 3^{-1 / 2} \int^{r} d r^{\prime} \frac{e^{r^{\prime}}}{\sqrt{1+\frac{2^{4 / 3}}{3 E \beta} e^{4 r^{\prime} / 3}}} \\
& = \pm 2^{-1 / 6} 3^{-1 / 2} e^{r} F\left(\frac{1}{2}, \frac{3}{4} ; \frac{7}{4},-\frac{2^{4 / 3}}{3 E \beta} e^{4 r / 3}\right),
\end{aligned}
$$


where $F(a, b ; c, z)$ is the hypergeometric function.

In two extreme regimes, Eq. (41) can be inverted. First consider the "slow-roll" limit when the brane is moving slowly and $E \beta<<1$. This corresponds to the limit when the kinetic energy of the brane is much smaller than the brane rest mass in the DBI action in Eq. (8). Using the identity

$$
F(a, b ; c, z)=(1-z)^{-a} F\left(a, c-b ; c, \frac{z}{z-1}\right),
$$

we obtain

$$
\tilde{\eta} \simeq \pm 3 \times 2^{-5 / 6} e^{r / 3} .
$$

Using Eq. (39) for $\tilde{v}_{\text {eff }}$ in the large $r$ limit, the potential reads

$$
\tilde{v}_{e f f} \simeq \frac{C \ln |\tilde{\eta}|}{\tilde{\eta}^{6}},
$$

where $C$ is an un-important coefficient (the reason will be clear later).

The other limit where Eq. (41) can be inverted is when $E \beta e^{4 r / 3}>>1$. This corresponds to the "fast-roll" limit, when the kinetic energy of the moving brane is comparable to the brane rest-mass. In this limit, using the identity given in Eq. (42) for hypergeometric functions, we obtain

$$
\tilde{\eta} \simeq 2^{-1 / 6} 3^{-1 / 2} e^{r}
$$

which in the large $r$ limit leads to

$$
\tilde{v}_{e f f} \simeq \frac{10}{9 \ln |\tilde{\eta}|} \frac{1}{\tilde{\eta}^{2}} .
$$

It is interesting that in the fast-roll case, the $\tilde{\eta}$-dependence of $\tilde{v}_{\text {eff }}$, up to a logarithmic correction, has the same form as in the inflationary cosmology. However, the constant prefactor is different: in the inflationary model the prefactor is 2 , while for the fast-roll case, it is 10/9. The constant has its origin in the asymptotic limit of the KS geometry, where it reaches pure AdS space. As is well known in the context of inflationary or Ekpyrotic cosmology, the constant 2 is crucial to obtain a scale-invariant spectrum starting with the Bunch-Davis vacuum initial conditions. This change in the coefficient results in a non-scaleinvariant prediction for the scalar spectral index $n_{s}$, as we will see explicitly in the next section. 
The effective potential $\tilde{v}_{\text {eff }}$ as a function of $\rho=(2 E \beta)^{1 / 2} \tilde{\eta}$ is given in Fig. 3. Suppose the brane starts from the UV region heading towards the IR region of the throat, so $\tilde{\eta}<0$

initially. The modes start in the sub-Hubble region where $-\tilde{k} \tilde{\eta}>>1$ and $\tilde{k}^{2}-\tilde{v}_{\text {eff }}>0$. They evolve as sub-horizon mode until "crossing the potential" (i.e. $\tilde{k}^{2}-\tilde{v}_{e f f}=0$ ) and then become super-Hubble. They remain super-Hubble until they cross the potential again near $r=0$ and become sub-Hubble. The evolution of the modes is sub-Hubble near the tip of the throat until the brane bounces back at $r=0$ or $\tilde{\eta}=0$. The mode evolution for $\tilde{\eta}>0$ is the mirror image of what happens for $\tilde{\eta}<0$.

The scalar spectral index can be calculated at an arbitrary point like $\tilde{\eta}_{*}$, as long as the mode is super-Hubble but in the decelerating phase at late times. One may identify $\tilde{\eta}=\tilde{\eta}_{*}$ with the surface of last scattering.

\section{SOLVING THE MODE EQUATION ANALYTICALLY}

Having obtained the effective potential $\tilde{v}_{\text {eff }}$ in the large $r$ limit of both fast-roll and slowroll cases, we can solve the mode equation and compute the spectrum of fluctuations using analytical approximations. First, recall that the scalar power spectral index $n_{s}$ is given by

$$
P_{\phi}(k) \equiv \frac{k^{3}}{2 \pi^{2}}\left|\phi_{k}\right|^{2} \sim k^{n_{s}-1}
$$

Let us start with the fast-roll case which is more amenable to analytic approximation and where the equations are more similar to those appearing in inflationary models. To solve the differential equation (37) analytically, we make a crude approximation here and neglect the logarithmic running in the effective potential (46). We simply replace the logarithm by one. Comparing with the exact result obtained numerically in the next section, we find that this is a reasonable approximation.

We wish to compute the power spectrum of $\phi_{k}$ at a late time $\eta_{*}$ on super-Hubble scales. Let us denote the time that the mode $k$ crosses the Hubble radius shortly after the bounce by $\eta_{2}(k)$, the time it enters the Hubble radius shortly before the bounce by $\eta_{1}(k)$, and the time it exits the Hubble radius during the initial contracting phase by $\eta_{0}(k)$. Due to the fact that the potential is very negative at the bounce point, in small $k$ limit, the $\mathrm{k}$-dependence of $\eta_{2}(k)$ and $\eta_{1}(k)$ is negligible. It is crucial to note that the negativity of the potential in the vicinity of the bounce point is generic as it was argued in the previous sections. Also, 
between $\eta_{1}(k)$ and $\eta_{2}(k)$, i.e. in the bounce region, the mode is oscillating and its amplitude does not change. This implies that as long as the potential around the bounce point is negative, the bounce region does not introduce any k-dependence in the transfer matrix. Hence, the power spectrum of $\phi_{k}$ at the late time $\eta_{*}$ has the same spectral index as the power spectrum at pre-bounce time $-\eta_{*}$ :

$$
P_{\phi}\left(k, \eta_{*}\right) \equiv k^{3}\left|\phi_{k}\left(\eta_{*}\right)\right|^{2}=k^{3} \mathcal{A}\left|\phi_{k}\left(-\eta_{*}\right)\right|^{2},
$$

where the amplification factor $\mathcal{A}$ is given by

$$
\mathcal{A}=\left(\frac{\left|\phi_{k}\left(\eta_{*}\right)\right|^{2}}{\left|\phi_{k}\left(\eta_{2}(k)\right)\right|^{2}}\right)\left(\frac{\left|\phi_{k}\left(\eta_{2}(k)\right)\right|^{2}}{\left|\phi_{k}\left(\eta_{1}(k)\right)\right|^{2}}\right)\left(\frac{\left|\phi_{k}\left(\eta_{1}(k)\right)\right|^{2}}{\left|\phi_{k}\left(-\eta_{*}(k)\right)\right|^{2}}\right)
$$

and is, to a first approximation, independent of $k$. Thus, the spectral shape is determined by the growth of the mode functions between initial Hubble radius crossing at $\eta_{0}(k)$ and the time $-\eta_{*}$ :

$$
P_{\phi}\left(k, \eta_{*}\right) \sim k^{3} \frac{\left|\phi_{k}\left(-\eta_{*}\right)\right|^{2}}{\left|\phi_{k}\left(\eta_{0}\right)\right|^{2}}\left|\phi_{k}\left(\eta_{0}\right)\right|^{2} .
$$

In the fast-roll case, the dominant solution of $\phi_{k}$ in the contracting phase is given by

$$
\phi_{k}(\eta) \sim \eta^{-2 / 3} .
$$

Since $\eta_{0}(k)$ is given by $\tilde{k}^{2}=\tilde{v}_{e f f}\left(\eta_{0}(k)\right)$ we have

$$
\eta_{0}(k) \sim k^{-1} .
$$

Inserting (51) and (52) into (50) and assuming that the modes start out on sub-Hubble scales during the period of contraction (i.e. for $\eta<\eta_{0}(k)$ ) in the vacuum state with the Bunch-Davis initial condition

$$
\phi_{k} \sim \frac{A}{\sqrt{2 k}} e^{-i \tilde{k} \tilde{\eta}}
$$

we obtain

$$
P_{\phi}(k) \sim k^{2 / 3},
$$

and thus the spectral index is predicted to be $n_{s}=5 / 3$.

To be slightly more precise, using the large $r$ limit of $\tilde{v}_{\text {eff }}$ form Eq. (46), the solution of (37) is given in terms of Hankel functions of first and second kind

$$
\phi_{k}=\sqrt{\tilde{\eta}}\left[A_{1} H_{7 / 6}{ }^{(1)}(-\tilde{k} \tilde{\eta})+A_{2} H_{7 / 6}{ }^{(2)}(-\tilde{k} \tilde{\eta})\right],
$$


where $A_{1}$ and $A_{2}$ are two constants of integration. Like in inflationary models, only the Hankel function of first type matches with the initial vacuum condition since

$$
H_{7 / 6}^{(1)}(x>>1) \sim \sqrt{\frac{2}{\pi x}} e^{i\left(x-\frac{10 \pi}{12}\right)} \quad, \quad H_{7 / 6}^{(2)}(x>>1) \sim \sqrt{\frac{2}{\pi x}} e^{-i\left(x-\frac{10 \pi}{12}\right)}
$$

which implies that $A_{2}=0$. Thus, we obtain the scaling of $\phi_{k}$ with $\eta$ given in (51), and, as shown above, this leads to

$$
n_{s}=5 / 3
$$

Interestingly enough, the spectrum is not scale invariant. As explained before, this is due to the numerical factor $10 / 9$ in Eq. (46) for $\tilde{v}_{\text {eff }}$. This particular number originated from the fact that the UV region of the throat is well-approximated by pure AdS geometry. This indicates that for pure AdS geometry, the spectral index will be $n_{s}=5 / 3$. As explained before, we have neglected the logarithmic correction into $\tilde{v}_{e f f}$, which originates from the logarithmic correction to the AdS geometry in the KS solution. Considering the logarithmic correction, our numerical analysis (presented in next section) show that $n_{s} \simeq 2.3$.

The case of slow-roll proved to be more involved. Even when we neglect the logarithmic correction for $\tilde{v}_{\text {eff }}$ in Eq. (44), we could not solve the differential equation (37) simultaneously for both sub-Hubble and super-Hubble modes. However, we can solve it for sub-Hubble and super-Hubble modes separately. For sub-Hubble modes the solution is as given by vacuum state Eq. (53). For super-Hubble modes, the solution of Eq. (37) with effective potential Eq. (44) is given in terms of Bessel and Neumann functions

$$
\phi_{k}=\sqrt{\tilde{\eta}}\left[\tilde{B}_{1} J_{-1 / 4}\left(\frac{\sqrt{-C}}{2 \tilde{\eta}^{2}}\right)+\tilde{B}_{2} Y_{-1 / 4}\left(\frac{\sqrt{-C}}{2 \tilde{\eta}^{2}}\right)\right] .
$$

At the transition $\tilde{\eta}_{0}(k)$ point when $\tilde{k}^{2}-\tilde{v}_{e f f}=0$, this solution should smoothly go over to the solution for the sub-Hubble modes. The transition point is given by

$$
\tilde{\eta}_{0}(k)=C^{1 / 2} k^{-1 / 3} .
$$

Since we are interested in large scale perturbations, $k<<1$, so $\tilde{\eta}_{0}(k)>>1$ and we can use the small argument approximation for the Bessel and Neumann functions

$$
J_{\nu}(x<<1) \sim x^{\nu} \quad, \quad Y_{\nu}(x<<1) \sim-x^{-\nu}
$$


Using these approximations in Eq. (58), we obtain

$$
\phi_{k}\left(\tilde{\eta}_{0}\right) \simeq\left(B_{1}+B_{2} \tilde{\eta}_{0}\right)
$$

where $B_{1}$ and $B_{2}$ are new constant of integrations related to $\tilde{B}_{1}$ and $\tilde{B}_{2}$. Matching this solution to the sub-Hubble solution Eq. (53) at $\tilde{\eta}_{0}(k)$, we find

$$
B_{1} \simeq \frac{A}{\sqrt{2 k}}\left(1+i k \tilde{\eta}_{0}(k)\right) e^{-i k \tilde{\eta}_{0}(k)} \quad, \quad B_{2} \simeq \frac{-i A}{\sqrt{2}} \sqrt{k} e^{-i k \tilde{\eta}_{0}(k)} .
$$

For large scale perturbations where $k \rightarrow 0, B_{1}$ scales like $k^{-1 / 2}$, while $B_{2}$ scales like $k^{1 / 2}$ and from Eq 61 we obtain $\phi_{k}\left(\tilde{\eta}_{0}\right) \sim k^{-1 / 2}$. Using this in Eq 50 , one obtains

$$
n_{s}=3
$$

We have calculated the spectral index numerically and found $n_{s}=3 \pm 10^{-5}$, in agreement with the above analytical value.

Indeed, one can argue that for potentials of the form $\tilde{v}_{\text {eff }} \sim \tilde{\eta}^{-n}$, with $n>2$, the spectral index is always equal to 3 . On the other hand, $n=2$ is the critical value where the spectral index crucially depends on the numerical coefficient in $\tilde{v}_{\text {eff }}$. For a potential of the form $\tilde{v}_{e f f}=c \tilde{\eta}^{-2}$, one can show that $n_{s}=4-\sqrt{1+4 c}$. For inflationary models in the limit where slow-roll corrections are ignored $c=2$ and one obtains $n_{s}=1$. In our fast-roll case $c=10 / 9$, which leads to $n_{s}=\frac{5}{3}$, as we have shown before.

\section{NUMERICS}

In this section we discuss our numerical results. In order to solve the equation of motion for $\phi_{k}$ (37) numerically, we find it more convenient to work with the coordinate $r$ related to $\tilde{\eta}$ through 40 . In this coordinate, we look for a left moving wave solution initialized at very large values of $r$ which eventually reflects back to the large $r$ region. The equation of motion is initialized with the Bunch-Davis vacuum initial data given in Eq (53). Boundary conditions at the origin of the $r$-coordinate are easily found to be the impact boundary conditions

$$
\begin{aligned}
\left.\phi_{\text {kin }}\right|_{r=0^{+}} & =\left.\phi_{\text {kout }}\right|_{r=0^{-}}, \\
\left.\frac{d \phi_{k \text { in }}}{d r}\right|_{r=0^{+}} & =-\left.\frac{d \phi_{k \text { out }}}{d r}\right|_{r=0^{-}},
\end{aligned}
$$



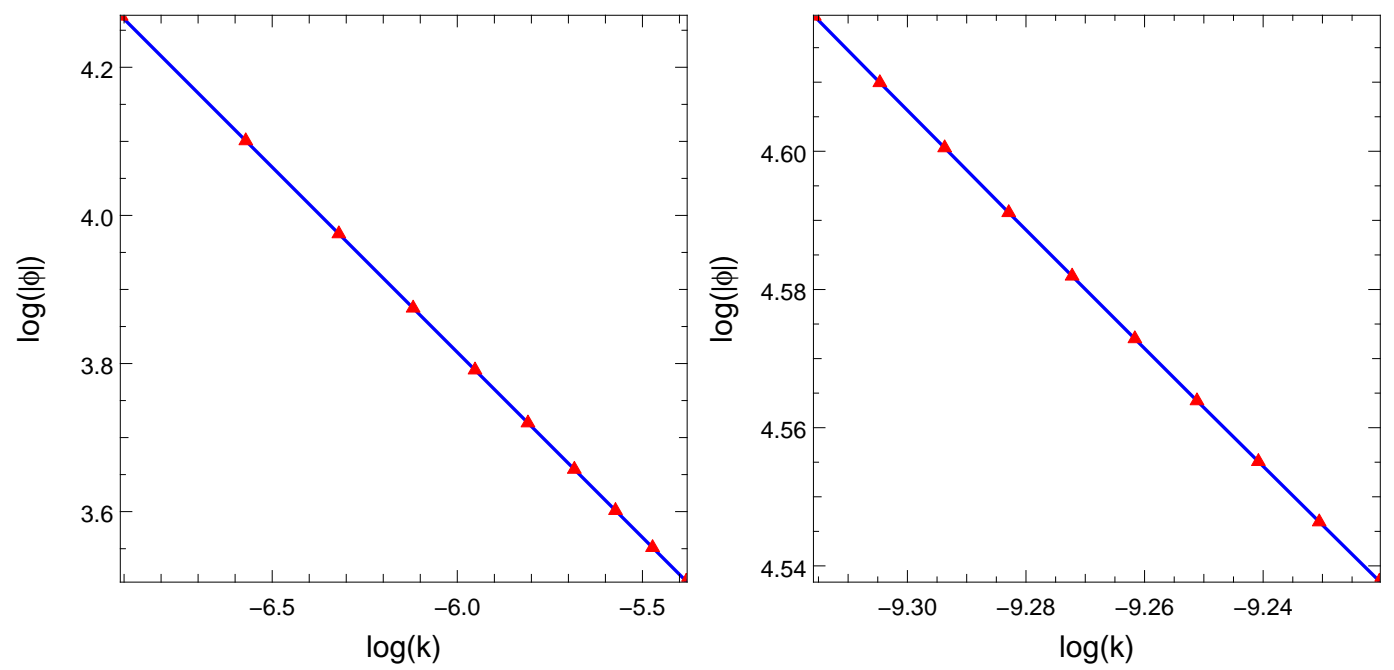

FIG. 4: The $k$-dependence of $\phi_{k}\left(\eta_{*}\right)$ is numerically plotted. The figure on the left corresponds to the slow-roll limit with the slope equal to -0.5 and $n_{s}=3$, while that of the right hand side corresponds to the fast-roll case with slope equal to -0.85 and $n_{s}=2.3$. The data points are shown by red triangles.

where $\phi_{k \text { in }}$ and $\phi_{k \text { out }}$ refer to the incoming and outgoing waves at the origin, respectively. The quantity of interest to us is the spectral index defined by (47). As usual, $\phi_{k}$ is evaluated at a convenient point in the super-Hubble region. It is worth emphasizing that picking a different point will only amount to a change in the amplitude of the power spectrum and will not alter its k-dependence or the index as long as it lies within the super-Hubble region. We solve (37) for different values of $k$ and read off the index from the log-log plot of $k^{3}\left|\phi_{k}\right|^{2}$ versus $k$, generated numerically for a range of $k$ values. We observe a negligible running of the index, for either small or large values of the parameter $E \beta$, as we dial $k$. We made sure that a change in the location where the initial Bunch-Davis vacuum condition was imposed on our numerical solution did not influence the resulting index. Fig. 4 illustrates our results for the index in two different limits for $E \beta$. The left side represents the results in the small $E \beta$ limit. The right side gives the result in the opposite limit where $E \beta$ is large (by which we simply mean the limit where the second term under the square root in 40 can be ignored). Note that the limit itself depends on the wavelength of the observed large scale structures, $k_{\text {obs }}^{-1}$, around which the index is calculated. 


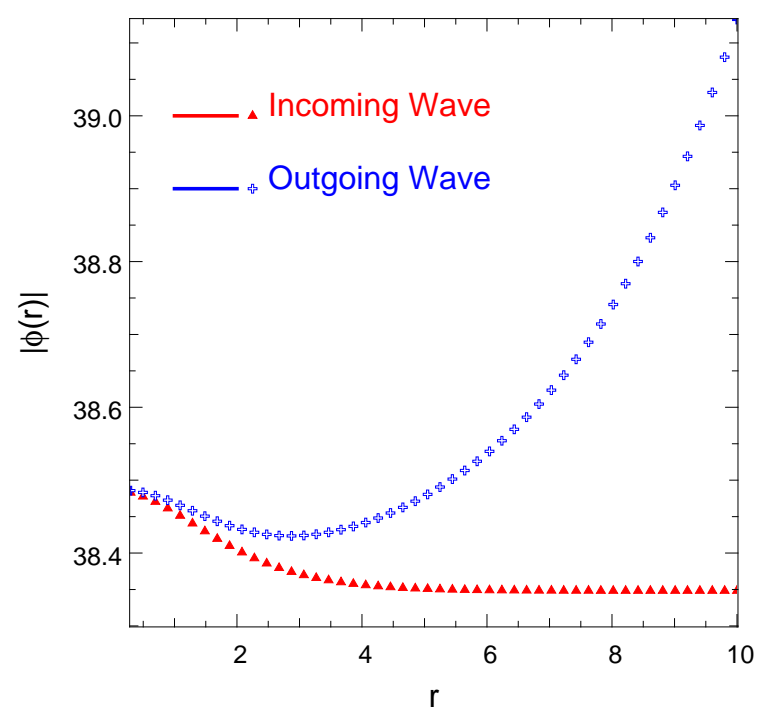

FIG. 5: The wave function is presented here. The red(blue) curve corresponds to contracting(expanding) phase. As time increase, i.e. $\tilde{\eta}$ increases, the wave function also increases. This indicates that the dominant mode in the contracting phase is matched to the dominant mode in the expanding phase.

Fig. 5 shows a sample wavefunction (its modulus to be exact) plotted for $\tilde{k}=0.0034$. The lower curve represents the wavefunction in the contracting phase. The amplitude is growing as the bounce point $r=0$ is approached. The upper curve is the amplitude of the wavefunction in the expanding phase. As is apparent, the amplitude is growing in time (i.e. as $\tilde{\eta}$ increases). This demonstrates explicitly that the dominant mode of $\phi_{k}$ in the contracting phase couples with un-suppressed amplitude to the dominant mode in the expanding phase, unlike what is obtained in models in which a contracting Einstein cosmology is matched to an expanding Einstein cosmology at a singular hypersurface making use of the usual matching conditions of [16, 17].

Note that in the simulation of Fig. 4, the initial condition was imposed at $r=10$, where the brane is deep in the UV region, well-approximated by the AdS geometry.

\section{BACKREACTION}

As discussed in [25], we need to check that the gravitational instability associated with long wavelength gravitational perturbations does not destabilize the homogeneous back- 
ground. The length scale associated with this instability is the Jeans length given by $L_{J}=v_{s} / \sqrt{G \rho}$ where $v_{s}$ is the speed of sound, $G$ is the Newton's constant and $\rho$ is the energy density on the brane. The time scale associated with this instability is $t_{i n s} \geq L_{J}$.

For our model, from Eq. (8), we obtain

$$
\begin{aligned}
\rho & =T_{3} h^{-1}\left(1-\sqrt{1-g h^{1 / 2} \dot{r}^{2}}\right) \\
& =\frac{E T_{3}}{1+E h} .
\end{aligned}
$$

In the slow-roll limit, one can see that $\rho \simeq E T_{3}$ is constant.

Using $G \leq g_{s}^{2} l_{s}^{2}$ for our compactification [25], we have

$$
t_{\text {ins }} \geq L_{J}=\frac{v_{s}}{\sqrt{G \rho}} \geq v_{s} l_{s} \sqrt{\frac{1+E h}{E}} .
$$

Suppose one releases the brane from the point $r=r_{*}$ with the initial velocity $v_{*}$. Since the brane is accelerating towards the tip of the throat, the time for the bounce satisfies

$$
t_{\text {bounce }} \leq \frac{2 d_{*}}{v_{*}}
$$

where $d_{*}$ is the physical distance traveled by the brane before reaching the bottom of the throat. One can show that

$$
v_{*}=\sqrt{E h\left(r_{*}\right)\left(2+E h\left(r_{*}\right)\right)}
$$

Discarding factors of order unity, taking $v_{s} \sim 1$ and using the fact that $\epsilon^{4 / 3} e^{2 r_{*} / 3} \sim l_{s}^{2}$ one can show

$$
\frac{t_{\text {bounce }}}{t_{\text {ins }}}<\frac{r_{*}^{3 / 4}}{\sqrt{g_{s} M}}\left[\left(1+E h\left(r_{*}\right)\right)\left(2+E h\left(r_{*}\right)\right)\right]^{-1 / 2} .
$$

In the slow-roll limit when $E h\left(r_{*}\right)<<1$, one reaches the bound obtained in [25]. We see that this bound gets even weaker for the fast-roll limit, when $E h\left(r_{*}\right)>>1$. As an estimate, one can take $r_{*} \sim(5-10)$, when the brane is deep inside the throat. Also, to trust the supergravity limit, we take $g_{s} M>>1$. In this limit it is thus justified to neglect the effect of gravitational instability on the brane.

\section{DISCUSSION}

We have studied the evolution of cosmological fluctuations in a mirage cosmology setup in which the observer lives on a BPS D3-brane which is moving into and out of a KlebanovStrassler throat. This provides a simple model of a non-singular bouncing cosmology. 
Our main results are twofold. First, we find that the growing mode of the cosmological fluctuations in the contracting phase couples without suppression to the growing mode in the expanding phase. This is unlike what happens in a setup in which the fluctuations are treated in Einstein gravity in both the expanding and contracting phase, and then matched through a singular hypersurface using the analog of the Israel matching conditions. Negativity of the effective potential felt by the perturbations in the neighborhood of the bounce point appears to be generic in the models of the kind studied in this paper. As we saw, this leads to the fact that the bounce region does not contribute any $k$-dependence to the spectral index. Secondly, we find that if we set off the modes in their Bunch-Davies vacuum on subHubble scales in the contracting phase, a final spectrum in the expanding phase which is not consistent with observations will emerge. The specific spectral index depends on whether the brane is moving fast or slow. In the latter case, the resulting spectral index is $n_{s}=3$, in the former $n_{s}$ is closer to 2 but still completely inconsistent with the data which demands $n_{s}=0.95 \pm 0.05$.

In this analysis we did not take into account the effects of volume modulus stabilization, such as can be achieved by wrapped D7-branes, on the mobile D3-brane. In a realistic model where all back-reactions are included, the brane feel an attractive force towards the tip of the throat [36, 37]. This changes $v_{\text {eff }}$ and it is interesting to see whether or not these effects can modify the spectral index. It is also possible that mirage cosmology motion in a different type of throat might lead to a spectrum consistent with the cosmological data. It is also possible that a pre-cursor phase which involves extra physics (as assumed e.g. in the Ekpyrotic scenario) leads to a consistent spectrum. Finally, it is possible that, if the bounce phase lasts sufficiently long, thermal fluctuations of a gas of closed strings with winding modes will generate a scale-invariant spectrum [38, 39, 40].

\section{Acknowledgments}

We thank D. Easson, C. Germani, N. Grandi, J. Khoury, B. Ovrut, D. Steer and A. Tolley for useful discussions and K. Hassani for computer assistance. This work is supported by NSERC under the Discovery Grant program, by Canada Research Chair funds (RB), and by funds from a FQRNT Team Grant. O.S. is supported in part by a McGill Tomlinson Postdoctoral Fellowship. 


\section{APPENDIX A: LINEAR EQUATION OF MOTION}

Here we present the details of the calculations leading to the linear perturbation equation 23). Although we perform the analysis for a D3-brane moving in a five dimensional background but the result is applicable to a Dp-brane with co-dimension one, i.e. $p=D-1$, where $D$ is the background space-time dimension.

As was briefly described in Section 2, at each point in space-time we can choose the normal vector base $\left\{e_{a}, n\right\}$, such that

$$
e_{a}^{M}=\frac{\partial X^{M}}{\partial \xi^{a}} \equiv X_{, a}^{M} \quad, \quad \gamma_{a b}=G\left(e_{a}, e_{b}\right)
$$

and

$$
G\left(n, e_{a}\right)=0 \quad, \quad G(n, n)=1
$$

where $G(u, v) \equiv G_{M N} u^{M} v^{N}$.

The following projection relation also holds:

$$
\gamma^{a b} X_{, a}^{M} X_{, b}^{N}=g^{M N}-n^{M} n^{N}
$$

We are interested in the normal displacements of the brane

$$
X^{M}=\bar{X}^{M}+\Phi n^{M}
$$

Under this transformation, we have

$$
\begin{aligned}
\delta \gamma_{a b} & =\Phi\left(G_{M N, P} n^{P} X_{, a}^{M} X_{, b}^{N}+2 G_{M N} n_{, a}^{M} X_{, b}^{N}\right) \\
& =2 K_{a b} \Phi
\end{aligned}
$$

where $K_{a b}$ is the extrinsic curvature of the surface defined in Eq. 22 ).

The equation of motion for the normal displacement $\Phi$ from the action $(20)$ is obtained by

$$
\begin{aligned}
\delta_{\Phi} S & =-T_{p} \int d^{p} \xi\left(\frac{1}{2} \sqrt{-|\gamma|} \gamma^{a b} \delta \gamma_{a b}-\frac{\partial X^{0}}{\partial t} C_{(4), M} \delta X^{M}-C_{(4)} \frac{\partial \delta X^{0}}{\partial t}\right) \\
& =-T_{p} \int d^{p} \xi\left[\sqrt{-|\gamma|} K-\frac{\partial X^{0}}{\partial t} C_{(4), M} n^{M}+n^{0} \frac{\partial C_{(4)}}{\partial t}\right] \Phi,
\end{aligned}
$$


which produces Eq. (21).

To get the linearized equation of motion we perturb Eq. (21) around Eq. (A4). The perturbed equation of motion is to some extent involved. Here we present the outline of the calculations. The linear equation is obtained by perturbing the left hand side (LHS) and right hand side (RHS) of Eq. (21) separately.

For the LHS, we have

$$
\delta(L H S)=\delta(\sqrt{-|\gamma|} K)=\sqrt{-|\gamma|}\left[\left(K^{2}-2 K^{a b} K_{a b}\right) \Phi+\gamma^{a b} \delta K_{a b}\right] .
$$

To obtain the terms proportional to $\Phi$, the relation (A5) for $\delta \gamma_{a b}$ was used. To calculate $\delta K_{a b}$, it is very useful to perform the analysis in locally Gaussian normal coordinates (GNC), where at each point $G_{M N, P}=\Gamma_{Q S}^{P}=0$, where $\Gamma_{Q S}^{P}$ is the connection compatible with the metric $G_{M N}$. Starting with the expression for $K_{a b}$ given in Eq. (A5), in GNC we have

$$
\gamma^{a b} \delta K_{a b}=\frac{\Phi}{2} n^{P} n^{Q}\left(G^{M N}-n^{M} n^{N}\right) G_{M N, P Q}+\gamma^{a b} G_{M N}\left(\delta X_{, b}^{M} n_{, a}^{M}+X_{, b}^{N} \delta n_{, a}^{M}\right)
$$

where to obtain the terms proportional to $\Phi$ the projection identity Eq. (A3) was used. We now calculate the last two terms in Eq. A8 separately. To do that we note that

$$
\delta n^{M}=A^{a} e_{a}^{M}+B n^{M}
$$

where

$$
\begin{aligned}
B & =-\frac{\Phi}{2} G_{M N, P} n^{M} n^{N} n^{P} \\
A^{a} & =-\gamma^{a b} \Phi_{, b}-\gamma^{a b}\left(G_{M N, P} X_{, b}^{N} n^{M} n^{P}+G_{M N} n^{M} n_{, b}^{N}\right) \Phi .
\end{aligned}
$$

Using this for the third term in Eq. (A8) we obtain

$$
\begin{aligned}
\gamma^{a b} G_{M N} \delta X_{, b}^{M} n_{, a}^{M} & =\gamma^{a b} G_{M N} n_{, a}^{M}\left(n_{, b}^{N} \Phi+n^{N} \Phi_{, b}\right) \\
& =\gamma^{a b} G_{M N} n_{, a}^{M} n_{, b}^{N} \Phi \\
& =\Phi K^{a b} K_{a b} .
\end{aligned}
$$

To go from the first line to the second line, the relation $n^{M} n_{M, a}=0$ which holds in GNC was used. The final identity in the above equation is easy to prove in GNC following a chain identity for partial derivatives. 
Similarly for the last term in Eq. A8) we have

$$
\begin{aligned}
\gamma^{a b} G_{M N} X_{, b}^{N} \delta n_{, a}^{M} & =\gamma^{a b} G_{M N}\left(X_{, c}^{M} X_{, b}^{N} A_{, b}^{c}+X_{, b}^{N} X_{, a c}^{M} A^{c}\right) \\
& =A_{, a}^{a}+A^{c} \gamma^{a b} G_{M N} X_{, b}^{N} X_{, a c}^{M}
\end{aligned}
$$

One notes that the term containing $B$ vanishes in GNC while the term containing $B_{, a}$ vanishes due to relation $G\left(n, e_{a}\right)=0$.

After some algebra, and working in GNC, one can show that

$$
\begin{aligned}
A_{, a}^{a} & =-\left[\left(G^{P N}-n^{P} n^{N}\right) n^{M} n^{Q} G_{M N, P Q}+\gamma^{a c} G_{M N} n^{M} n_{, a c}^{N}+K^{a b} K_{a b}\right] \Phi \\
& -\gamma^{a c} \Phi_{, a c}-\gamma_{, c}^{a c} \Phi_{, a}
\end{aligned}
$$

and

$$
A^{c} \gamma^{a b} G_{M N} X_{, b}^{N} X_{, a c}^{M}=-\gamma^{a b} \gamma^{c d} G_{M N} X_{, b}^{N} X_{, a c}^{M} \Phi_{, d}
$$

Combining all the terms, we obtain

$$
\begin{aligned}
\gamma^{a b} G_{M N} X_{, b}^{N} \delta n_{, a}^{M}= & -\nabla^{2} \Phi-K^{a b} K_{a b} \Phi-\gamma^{a c} G_{M N} n^{M} n_{, a c}^{N} \Phi \\
& -\left(G^{P N}-n^{P} n^{N}\right) n^{M} n^{Q} G_{M N, P Q} \Phi
\end{aligned}
$$

where $\nabla^{2}$ is the Laplacian defined by the metric $\gamma_{a b}$.

Combining Eqs. A15), A11 and A8) we obtain

$$
\begin{aligned}
\gamma^{a b} \delta K_{a b}= & -\nabla^{2} \Phi+\frac{1}{2} n^{P} n^{Q} g^{M N}\left(g_{M N, P Q}-2 g_{N P, M Q}\right) \Phi \\
& +n^{M} n^{N} n^{P} n^{Q} g_{P Q, M N} \Phi-\gamma^{a b} G_{M N} n^{M} n_{, a b}^{N} \Phi .
\end{aligned}
$$

We need to get rid of the last term above containing second derivatives of $n$. To do this, we take derivatives of the identity $G(n, n)=1$ in GNC, which leads to

$$
\gamma^{a b} G_{M N} n^{M} n_{, a b}^{N}=-\frac{1}{2} n^{M} n^{N}\left(G^{P Q}-n^{P} n^{Q}\right) G_{M N, P Q}-K^{a b} K_{a b}
$$

Using the above expression in Eq. A16), we have

$$
\gamma^{a b} \delta K_{a b}=-\nabla^{2} \Phi+K^{a b} K_{a b}-R_{M N} n^{M} n^{N}
$$

where $R_{M N}$ is the Ricci tensor of the background geometry. 
Thus, our final expression for $\delta(L H S)$ in Eq. A7) is

$$
\delta(L H S)=\sqrt{-|\gamma|}\left[-\nabla^{2} \Phi+\left(K^{2}-K^{a b} K_{a b}-R_{M N} n^{M} n^{N}\right) \Phi\right]
$$

in agreement with [35].

For the RHS of Eq. (21) we have

$$
\delta(R H S)=\left[C_{(4)}^{\prime \prime} n^{r}\left(n^{r}-\dot{R} n^{0}\right)+C_{(4)}^{\prime}\left(n^{r} \dot{n}^{0}-n^{0} \dot{n}^{r}\right)\right] \Phi+C_{(4)}^{\prime}\left(\delta n^{r}-\dot{R} \delta n^{0}\right) \Phi .
$$

Using the formula $(\mathrm{A} 9$ for $\delta n$, one can show that

$$
\begin{aligned}
\delta n^{r}-\dot{R} \delta n^{0} & =\frac{\left(G_{00}+G_{r r} \dot{R}^{2}\right) n^{r}}{2 G_{00} g_{r r}\left(n^{0} \dot{R}-n^{r}\right)}\left(G_{00}^{\prime}\left(n^{0}\right)^{2}+G_{r r}^{\prime}\left(n^{r}\right)^{2}\right) \Phi \\
& =\frac{n^{r}}{\dot{R}}\left(\dot{n}^{r}-\dot{R} \dot{n}^{0}\right)
\end{aligned}
$$

where to get the final line the relations $G(n, n)=1$ and $G\left(n, e_{a}\right)=0$ were used.

Combining Eqs. A21 and A20 we obtain

$$
\delta(R H S)=\left(\frac{n^{r}}{\dot{R}}-n^{0}\right)\left(C_{(4)}^{\prime} n^{r}\right)_{, t} \Phi .
$$

Combining Eqs. (A22) and (A19), the desired linear equation of motion from Eq. (21) is

$$
\nabla^{2} \Phi+\left[K_{a b} K^{a b}+R_{M N} n^{M} n^{N}-K^{2}-\frac{1}{\sqrt{-|\gamma|}}\left(n^{0}-\frac{n^{r}}{\dot{R}}\right)\left(C_{(4)}^{\prime} n^{r}\right)_{, t}\right] \Phi=0 .
$$

\section{APPENDIX B: APPROXIMATIONS FOR $I(r)$ AND $K(r)$}

The following approximation formulae for the KS background are useful:

$$
\begin{aligned}
K(r \rightarrow 0) & \rightarrow(2 / 3)^{1 / 3}+\mathcal{O}\left(r^{2}\right) \\
K(r \rightarrow \infty) & \rightarrow 2^{1 / 3} e^{-r / 3} \\
I(r \rightarrow 0) & \rightarrow 0.72+\mathcal{O}\left(r^{2}\right) \\
I(r \rightarrow \infty) & \rightarrow 3.2^{-1 / 3}\left(r-\frac{1}{4}\right) e^{-4 r / 3}
\end{aligned}
$$

\section{REFERENCES}

[1] D. N. Spergel et al., "Wilkinson Microwave Anisotropy Probe (WMAP) three year results: Implications for cosmology," astro-ph/0603449; W. J. Percival et al. [The 2dFGRS Collabora- 
tion], "The 2dF Galaxy Redshift Survey: The power spectrum and the matter content of the universe," Mon. Not. Roy. Astron. Soc. 327, 1297 (2001), astro-ph/0105252; C. Stoughton et al. [SDSS Collaboration], "The Sloan Digital Sky Survey: Early data release," Astron. J. 123, 485 (2002); C. L. Bennett et al., "First Year Wilkinson Microwave Anisotropy Probe (WMAP) Observations: Preliminary Maps and Basic Results," Astrophys. J. Suppl. 148, 1 (2003), astro-ph/0302207.

[2] A. H. Guth, "The Inflationary Universe: A Possible Solution To The Horizon And Flatness Problems," Phys. Rev. D 23, 347 (1981); A. D. Linde, "A New Inflationary Universe Scenario: A Possible Solution Of The Horizon, Flatness, Homogeneity, Isotropy And Primordial Monopole Problems," Phys. Lett. B 108, 389 (1982); A. Albrecht and P. J. Steinhardt, "Cosmology For Grand Unified Theories With Radiatively Induced Symmetry Breaking," Phys. Rev. Lett. 48, 1220 (1982); K. Sato, "First Order Phase Transition Of A Vacuum And Expansion Of The Universe," Mon. Not. Roy. Astron. Soc. 195, 467 (1981); R. Brout, F. Englert and E. Gunzig, "The Creation Of The Universe As A Quantum Phenomenon," Annals Phys. 115, 78 (1978); A. A. Starobinsky, "A New Type Of Isotropic Cosmological Models Without Singularity," Phys. Lett. B 91, 99 (1980).

[3] R. H. Brandenberger, "Inflationary cosmology: Progress and problems," publ. in proc. of IPM School On Cosmology 1999: Large Scale Structure Formation, hep-ph/9910410.

[4] A. Borde and A. Vilenkin, "Eternal inflation and the initial singularity," Phys. Rev. Lett. 72, 3305 (1994) gr-qc/9312022.

[5] R. H. Brandenberger and J. Martin, "The robustness of inflation to changes in superPlanck-scale physics," Mod. Phys. Lett. A 16, 999 (2001), astro-ph/0005432; J. Martin and R. H. Brandenberger, "The trans-Planckian problem of inflationary cosmology," Phys. Rev. D 63, 123501 (2001), hep-th/0005209.

[6] R. Kallosh, L. Kofman and A. D. Linde, "Pyrotechnic universe," Phys. Rev. D 64, 123523 (2001), hep-th/0104073.

[7] M. Gasperini and G. Veneziano, "Pre - big bang in string cosmology" Astropart. Phys. 1, 317 (1993) hep-th/9211021; M. Gasperini and G. Veneziano, "The pre-big bang scenario in string cosmology," Phys. Rept. 373, 1 (2003) hep-th/0207130; J. E. Lidsey, D. Wands and E. J. Copeland, "Superstring cosmology," Phys. Rept. 337, 343 (2000) hep-th/9909061.

[8] J. Khoury, B. A. Ovrut, P. J. Steinhardt and N. Turok, "The ekpyrotic universe: Colliding 
branes and the origin of the hot big bang," Phys. Rev. D 64, 123522 (2001), hep-th/0103239.

[9] E. I. Buchbinder, J. Khoury and B. A. Ovrut, "New ekpyrotic cosmology" hep-th/0702154; P. Creminelli and L. Senatore, "A smooth bouncing cosmology with scale invariant spectrum," hep-th/0702165.

[10] J. Martin, P. Peter, N. Pinto Neto and D. J. Schwarz, "Passing through the bounce in the ekpyrotic models," Phys. Rev. D 65, 123513 (2002), hep-th/0112128.

[11] Y. F. Cai, T. Qiu, Y. S. Piao, M. Li and X. Zhang, "Bouncing Universe with Quintom Matter," 0704.1090 [gr-qc].

[12] L. R. Abramo and P. Peter, "K-Bounce," 0705.2893 [astro-ph].

[13] S. Tsujikawa, R. Brandenberger and F. Finelli, "On the construction of nonsingular prebig-bang and ekpyrotic cosmologies and the resulting density perturbations," Phys. Rev. D 66, 083513 (2002), hep-th/0207228, C. Cartier, J. c. Hwang and E. J. Copeland, "Evolution of cosmological perturbations in non-singular string cosmologies," Phys. Rev. D 64, 103504 (2001), astro-ph/0106197.

[14] T. Biswas, A. Mazumdar and W. Siegel, "Bouncing universes in string-inspired gravity," JCAP 0603, 009 (2006), hep-th/0508194.

[15] A. Kehagias and E. Kiritsis, "Mirage cosmology," JHEP 9911, 022 (1999), hep-th/9910174 S. H. S. Alexander, "On the varying speed of light in a brane-induced FRW universe," JHEP 0011, 017 (2000), hep-th/9912037.

[16] J. c. Hwang and E. T. Vishniac, "Gauge-invariant joining conditions for cosmological perturbations," Astrophys. J. 382, 363 (1991).

[17] N. Deruelle and V. F. Mukhanov, "On matching conditions for cosmological perturbations," Phys. Rev. D 52, 5549 (1995), gr-qc/9503050.

[18] W. Israel, "Singular hypersurfaces and thin shells in general relativity," Nuovo Cim. B 44S10, 1 (1966) [Erratum-ibid. B 48, 463 (1967 NUCIA,B44,1.1966)].

[19] R. Brustein, M. Gasperini, M. Giovannini, V. F. Mukhanov and G. Veneziano, "Metric perturbations in dilaton driven inflation," Phys. Rev. D 51, 6744 (1995), hep-th/9501066.

[20] D. H. Lyth, "The primordial curvature perturbation in the ekpyrotic universe," Phys. Lett. B 524, 1 (2002) hep-ph/0106153 D. H. Lyth, "The failure of cosmological perturbation theory in the new ekpyrotic scenario," Phys. Lett. B 526, 173 (2002), hep-ph/0110007; F. Finelli and R. Brandenberger, "On the spectrum of fluctuations in an effective field theory of the ekpy- 
rotic universe," JHEP 0111, 056 (2001), hep-th/0109004; J. c. Hwang, "Cosmological structure problem in the ekpyrotic scenario," Phys. Rev. D 65, 063514 (2002), astro-ph/0109045 J. Khoury, B. A. Ovrut, N. Seiberg, P. J. Steinhardt and N. Turok, "From big crunch to big bang," Phys. Rev. D 65, 086007 (2002), hep-th/0108187; P. Creminelli, A. Nicolis and M. Zaldarriaga, "Perturbations in bouncing cosmologies: Dynamical attractor vs scale invariance," Phys. Rev. D 71, 063505 (2005), hep-th/0411270.

[21] R. Durrer and F. Vernizzi, "Adiabatic perturbations in pre big bang models: Matching conditions and scale invariance," Phys. Rev. D 66, 083503 (2002), hep-ph/0203275.

[22] P. Peter and N. Pinto-Neto, "Primordial perturbations in a non singular bouncing universe model," Phys. Rev. D 66, 063509 (2002), hep-th/0203013; P. Peter, N. Pinto-Neto and D. A. Gonzalez, "Adiabatic and entropy perturbations propagation in a bouncing universe," JCAP 0312, 003 (2003), hep-th/0306005; J. Martin and P. Peter, "On the 'causality argument' in bouncing cosmologies," Phys. Rev. Lett. 92, 061301 (2004), astro-ph/0312488, J. Martin and P. Peter, "Parametric amplification of metric fluctuations through a bouncing phase," Phys. Rev. D 68, 103517 (2003), hep-th/0307077; J. Martin and P. Peter, "On the properties of the transition matrix in bouncing cosmologies," Phys. Rev. D 69, 107301 (2004), hep-th/0403173.

[23] S. Alexander, T. Biswas and R. Brandenberger, "On the Transfer of Adiabatic Fluctuations through a Nonsingular Cosmological Bounce", 0707.4679 [hep-th].

[24] F. Finelli, "Study of a class of four dimensional nonsingular cosmological bounces," JCAP 0310, 011 (2003), hep-th/0307068; L. E. Allen and D. Wands, "Cosmological perturbations through a simple bounce," Phys. Rev. D 70, 063515 (2004), astro-ph/0404441; V. Bozza and G. Veneziano, "Scalar perturbations in regular two-component bouncing cosmologies," Phys. Lett. B 625, 177 (2005), hep-th/0502047; V. Bozza and G. Veneziano, "Regular twocomponent bouncing cosmologies and perturbations therein," JCAP 0509, 007 (2005), grqc/0506040; M. Gasperini, M. Giovannini and G. Veneziano, "Perturbations in a non-singular bouncing universe," Phys. Lett. B 569, 113 (2003), hep-th/0306113.

[25] S. Kachru and L. McAllister, "Bouncing brane cosmologies from warped string compactifications," JHEP 0303, 018 (2003), hep-th/0205209.

[26] C. Germani, N. E. Grandi and A. Kehagias, "A stringy alternative to inflation: The cosmological slingshot scenario," arXiv:hep-th/0611246. 
[27] D. Easson, R. Gregory, G. Tasinato and I. Zavala, "Cycling in the throat," JHEP 0704, 026 (2007), hep-th/0701252.

[28] C. Germani, N. Grandi and A. Kehagias, "The Cosmological Slingshot Scenario: Myths and Facts," 0706.0023 [hep-th]; C. Germani and M. Liguori, "Matching WMAP 3-yrs results with the Cosmological Slingshot Primordial Spectrum," 0706.0025 [astro-ph].

[29] I. R. Klebanov and M. J. Strassler, "Supergravity and a confining gauge theory: Duality cascades and chi SB-resolution of naked singularities," JHEP 0008, 052 (2000), hep-th/0007191.

[30] C. P. Herzog, I. R. Klebanov and P. Ouyang, "Remarks on the warped deformed conifold," hep-th/0108101; C. P. Herzog, I. R. Klebanov and P. Ouyang, "D-branes on the conifold and $\mathrm{N}=1$ gauge / gravity dualities," hep-th/0205100.

[31] T. Boehm and D. A. Steer, "Perturbations on a moving D3-brane and mirage cosmology," Phys. Rev. D 66, 063510 (2002), hep-th/0206147.

[32] V. F. Mukhanov, H. A. Feldman and R. H. Brandenberger, "Theory of cosmological perturbations. Part 1. Classical perturbations. Part 2. Quantum theory of perturbations. Part 3. Extensions," Phys. Rept. 215, 203 (1992).

[33] R. H. Brandenberger, "Lectures on the theory of cosmological perturbations," Lect. Notes Phys. 646, 127 (2004), hep-th/0306071.

[34] J. M. Bardeen, "Gauge Invariant Cosmological Perturbations," Phys. Rev. D 22, 1882 (1980).

[35] J. Guven, "Covariant perturbations of domain walls in curved space-time," Phys. Rev. D 48, 4604 (1993), gr-qc/9304032, gr-qc/9304032.

[36] D. Baumann, A. Dymarsky, I. R. Klebanov, J. Maldacena, L. McAllister and A. Murugan, JHEP 0611, 031 (2006), hep-th/0607050.

[37] C. P. Burgess, J. M. Cline, K. Dasgupta and H. Firouzjahi, JHEP 0703, 027 (2007), hepth/0610320.

[38] A. Nayeri, R. H. Brandenberger and C. Vafa, "Producing a scale-invariant spectrum of perturbations in a Hagedorn phase of string cosmology," Phys. Rev. Lett. 97, 021302 (2006), hep-th/0511140.

[39] R. H. Brandenberger, A. Nayeri, S. P. Patil and C. Vafa, "String gas cosmology and structure formation," hep-th/0608121.

[40] R. H. Brandenberger, "String gas cosmology and structure formation: A brief review," hepth/0702001. 